\title{
Non-invasive diagnosis of a false left ventricular aneurysm by echocardiography and pulsed Doppler echocardiography
}

\author{
E GRUBE, D REDEL, R JANSON \\ From the Departments of Cardiology and Radiology and the Section of Pediatric Cardiology, \\ University of Bonn, West Germany
}

SUMMARY This report describes the diagnosis of a false left ventricular aneurysm by echocardiography and pulsed Doppler echocardiography. The patient successfully underwent resection of the pseudoaneurysm.

A diagnosis of a false left ventricular aneurysm and its differentiation from a true aneurysm is important for the treatment and prognosis of this uncommon disorder.

Pseudoaneurysms usually develop when blood perforates the left ventricular wall forming a chamber contained by blood, thrombotic material, and pericardial tissue. The main cause of false aneurysms is myocardial infarction (Hurst et al., 1963; van Tassel and Edwards, 1972). Unlike true aneurysms false aneurysms have a tendency to early and late rupture, necessitating early diagnosis and prompt surgical intervention (Vlodaver et al., 1975).

Echocardiography and nuclear medicine are wellrecognised diagnostic tools for detecting a pseudoaneurysm (Roelandt et al., 1975; Botvinick et al., 1976; van Mechelen et al., 1978). However, echocardiography is not able to differentiate the false aneurysm from a local haematoma, a pericardial cyst, a diverticulum, or a pericardial effusion, or from a true aneurysm. Only the demonstration of the small connection between the ventricular cavity and the aneurysm by angiocardiography could confirm the diagnosis.

In this report we describe a case of a large posteroinferolateral false aneurysm, which had been suspected by echocardiography and confirmed by angiocardiography, pulsed Doppler echocardiography, and cardiac surgery.

\section{Case report}

A 59-year-old man had been admitted to his local hospital in March 1974 with an inferior myocardial wall infarction.

This diagnosis was established by typical electrocardiographic and enzyme changes. Before this event he had never been in a hospital and he denied symptoms or signs suggestive of coronary artery disease. The patient's hospital course was uneventful and after 3 weeks he was discharged without medication. He was free of symptoms and did not see a doctor until May 1978 when he felt his heart beating irregularly. He was then admitted to our institution with a fast irregular heart rate between 80 and 120 beats/min and an arterial blood pressure of $170 / 100 \mathrm{mmHg}$. No peripheral oedema or venous congestion was noted, and no praecordial heave or apical impulse was felt.

On auscultation a fourth heart sound was heard but no other systolic or diastolic extra sound or murmur could be detected. The electrocardiogram showed atrial flutter with varying atrioventricular block. $Q$ waves were present in leads II, III, aVF, and V6, terminal negative $T$ waves in III and a VF, with ST segment elevation in II and III. This electrocardiogram was interpreted as showing an old inferolateral myocardial infarction and possible aneurysm.

The chest $x$-ray film showed dilatation of the heart. In the lateral view there was a large posterior bulge of the left ventricle with narrowing of the retrocardiac space.

After echocardiographic studies were performed, the patient underwent cardiac catheterisation with angiocardiography, and then successful cardiac surgery with resection of the pseduoaneurysm. 


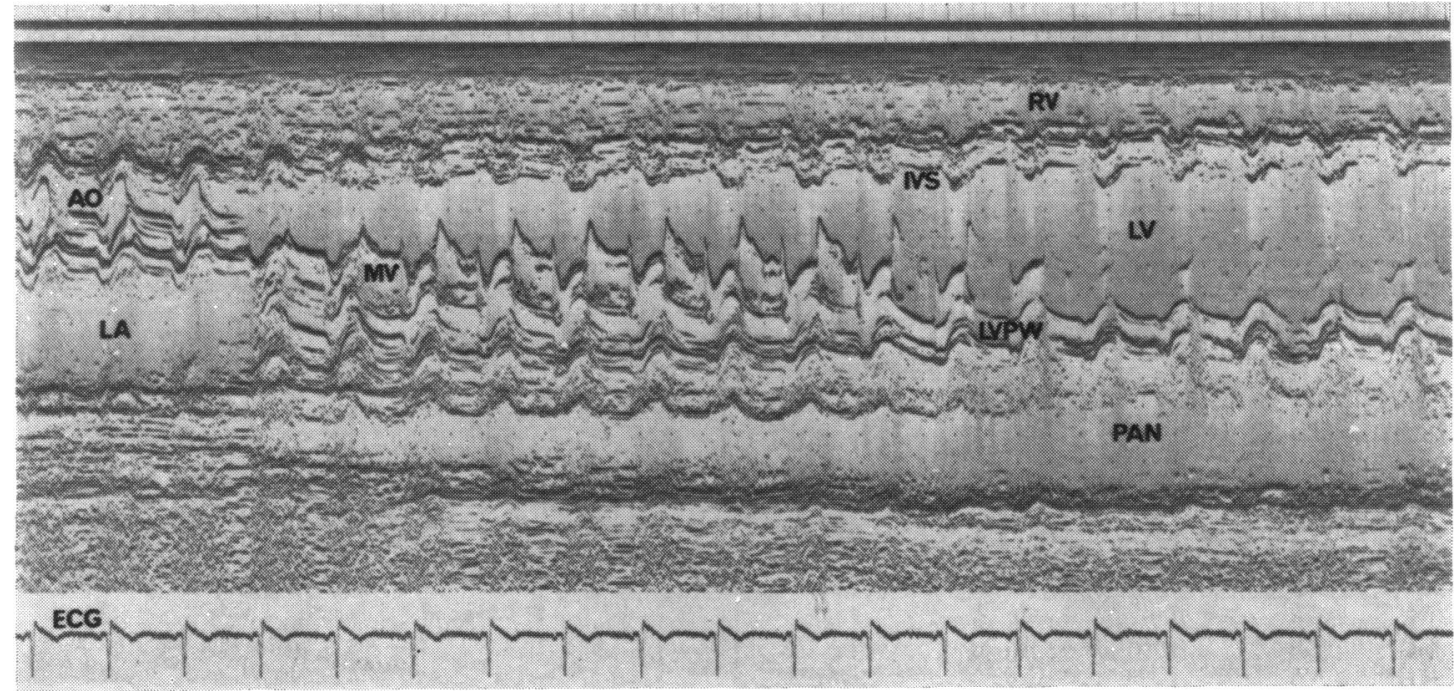

Fig. 1 Preoperative condensed $M$-mode scan from the aorta ( $A O)$ towards the left ventricle ( $L V)$. At the atrioventricular junction an echo-free area is recorded (PAN) behind the posterior left ventricular wall (LVPW) extending from the left atrium $(L A)$ to the left ventricle. $M V$, mitral valve; $R V$, right ventricle; IVS, interventricular septum; $P A N$, false or pseudoaneurysm.
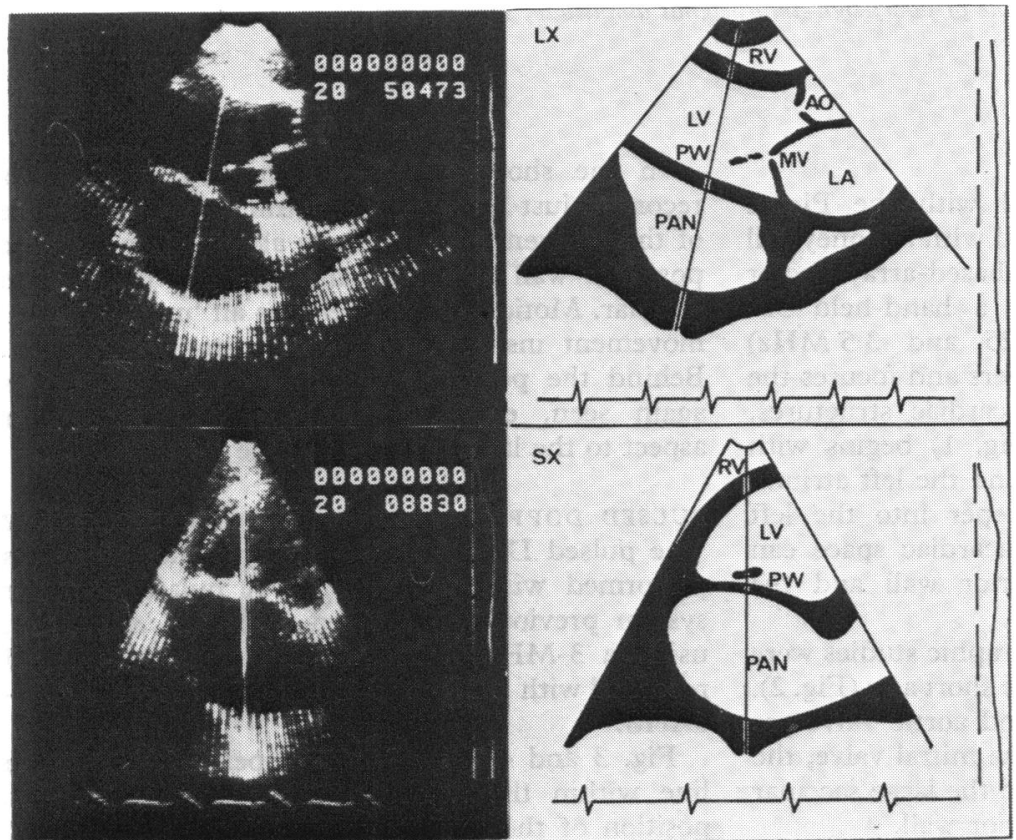

Fig. 2 Original two-dimensional echocardiographic pictures in long axis $(L X)$ and short axis $(S X)$ on the left, with drawings on the right. In the long axis plane, aorta $(A O)$, left atrum $(L A)$, mitral valve $(M V)$, and parts of the right $(R V)$ and left $(L V)$ ventricle are displayed simultaneously with the large saccular pseudoaneurysm (PAN) behind the posterior wall (PW) of the left ventricle. 


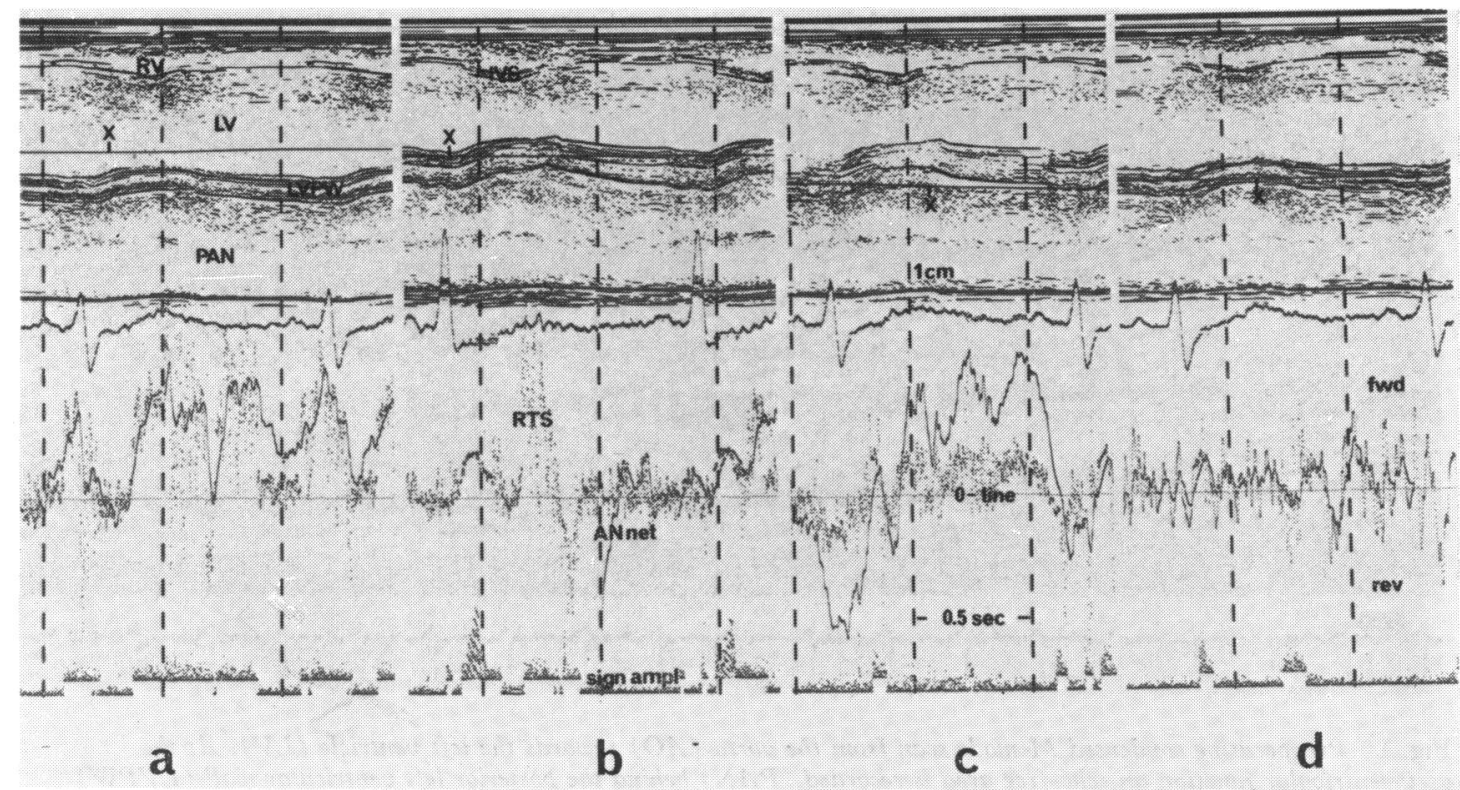

Fig. 3 M-mode display with depth line, indicating the position of the sample volume (upper half of the Fig.), electrocardiogram in the middle part, Doppler-shifted frequency print-outs in real-time spectral (RTS), and analogue-net (ANnet) analysis (lower half). (a) Highly disturbed flow in systole and diastole with great turbulence in the apical portion of the left ventricle (LV). (b) A short systolic highly turbulent flow inside the left ventricular posterior wall $(L V P W)$, marking the small communication defect. (c) The same disturbed flow pattern behind the posterior wall (LVPW) in the cavity of the pseudoaneurysm (PAN) as in the left ventricle (LV). (d) Only wall movement, no flow signals inside the normal left ventricular posterior wall (LVPW) M-mode abbreviations the same as in Fig. 1. Turbulent flow can be recognised best in the real-time spectral analysis print-out (RTS), while analogue-net curve (ANnet) shows more clearly the mean flow characteristics, that is the net-vector of forward ( $f w d)$ and reverse (rev) flow in every moment. O-line, zero-line of flow; sign ampl, signal amplitude, showing the strength of the flow signal going into the spectral processor.

\section{ECHOCARDIOGRAPHIC STUDY}

These studies were performed with the Picker Echoview-80-C system interfaced with a Honeywell Visicorder 1856 and an $80^{\circ}$ phased-array sector scanner Roche RT 400 using a hand-held 32element double-frequency $(2 \cdot 25$ and $3.5 \mathrm{MHz})$ transducer that electronically steers and focuses the ultrasound beam through the cardiac structures. A condensed $\mathrm{M}$-mode scan (Fig. 1) begins with the demonstration of the aorta and the left atrium. As the sound beam passes deeper into the left ventricle a large echo-free extracardiac space can be recorded behind the posterior wall and the mitral valve area.

Two-dimensional echocardiographic studies were performed in the long axis and the short axis (Fig. 2). In the long axis image, aorta and aortic valve are displayed simultaneously with the mitral valve, the left atrium, the left ventricle, and the large saccular echo-free area behind the posterior wall.
In the short axis image the left ventricle is recorded just beneath the mitral valve. The shape of the left ventricle is grossly abnormal in that the posterior wall is fibrotic and flattened rather than circular. Motion of this area was an 'up-and-down' movement instead of a co-ordinated contraction. Behind the posterior wall the echo-free space is again seen, extending from the inferoposterior aspect to the lateral portion of the left ventricle.

PULSED DOPPLER ECHOCARDIOGRAPHIC STUDY The pulsed Doppler echocardiographic study was performed with an ATL $500 \mathrm{~A}$ pulsed Doppler system previously described (Baker et al., 1977), using a $3-\mathrm{MHz}$ transducer focused at $7 \mathrm{~cm}$ and recorded with a Honeywell fibreoptic recorder LS6AHS.

Fig. 3 and 4 show in the upper half the depth line within the $M$-mode display, indicating the position of the sample volume. In the lower half 
print-outs of the Doppler-shifted frequency spectrum (real-time spectral analysis, analogue-net curve, and signal-amplitude trace) are shown simultaneously with the electrocardiogram. In the apical portion of the left ventricular cavity a highly disturbed flow pattern with turbulence in systole and diastole is displayed (Fig. 3a).

Moving the sample volume into the posterior wall of the left ventricle (Fig. 3b), a short systolic, highly turbulent flow appears on the RTS print-out. This obviously marks the site of communication between the left ventricle and the pseudoaneurysm.

As the sample volume is advanced into the cavity of the pseudoaneurysm almost the same systolicdiastolic flow pattern as in the ventricular cavity is found (Fig. 3c). This indicates a free communication between both cavities. In Fig. 3d the sample volume is positioned in the normal left ventricular posterior wall where only some wall motion but no flow signals can be obtained. Fig. 4 shows a continuous frequency print-out as the sample volume is moved uninterruptedly from the cavity of the pseudoaneurysm (on the left) through the communication site of the posterior wall into the left ventricular cavity (on the right).
Blood flow signals can be shown again in both cavities and followed through the wall, thus marking the site of perforation and communication. In the posterior part of the false aneurysm no flow signals can be detected, indicating that the defect is very small, therefore not allowing propagation of left ventricular flow deeper into the cavity.

\section{Discussion}

Perforation of an infarcted area may result either in a spontaneous rupture with fatal outcome in the early stage of a myocardial infarction or in a slow progressive leakage of blood into the pericardial sac.

If there is partial adherence between pericardial and epicardial layers a local haematoma will be formed. This would be compatible with life (Gueron et al., 1975). Diagnosis of pseudoaneurysms and their differentiation from true aneurysms is very important because of the tendency of false aneurysms to rupture even at a late stage (Vlodaver et al., 1975). If diagnosed early enough surgery has proved successful (Davidson et al., 1977).

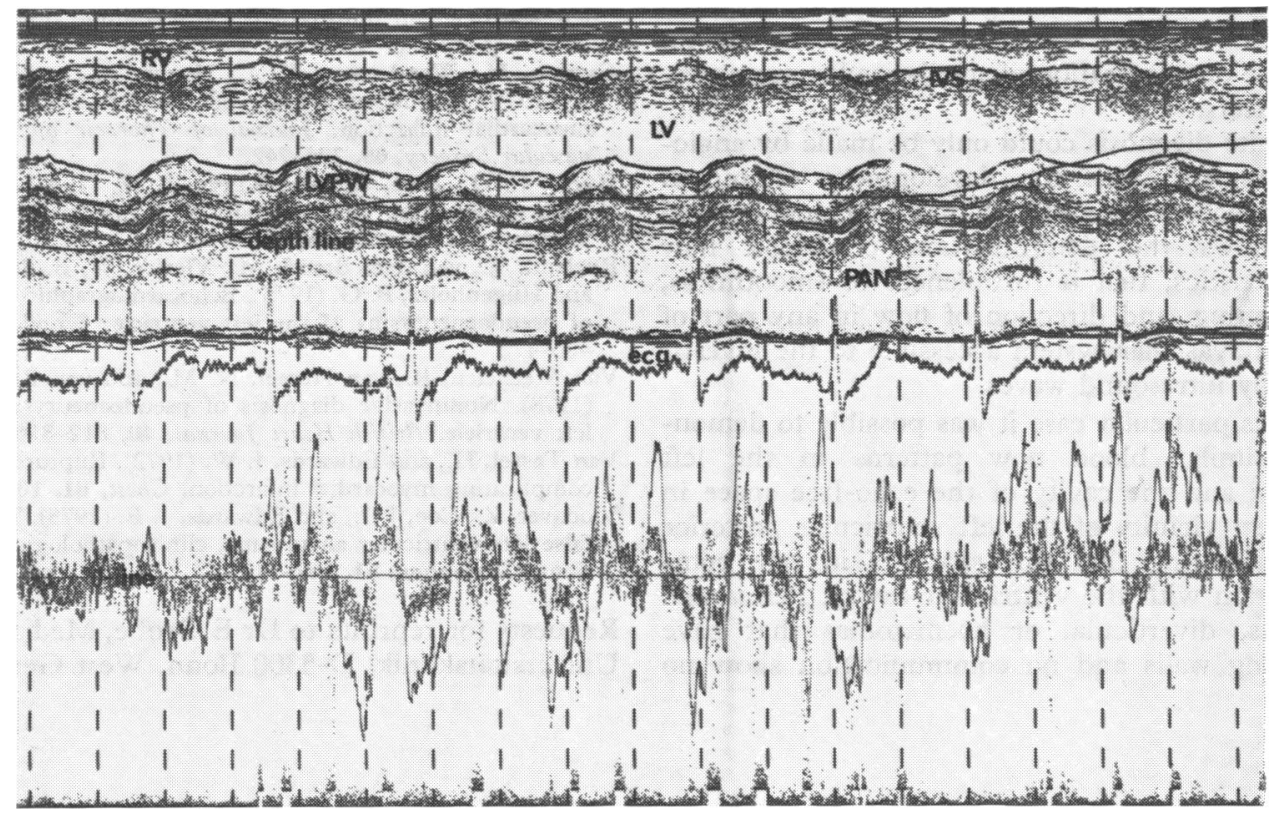

Fig. 4 Frequency print-out of the continuous sample volume movement (depth line) from the anterior third of the cavity of the pseudoaneurysm (PAN) (left) to the direct vicinity of the left ventricular posterior wall (LVPW) (middle), and across it into the left ventricular cavity (LV) (right). No flow signals are found in the deeper part of the pseudoaneurysm; near the posterior wall there is systolic and diastolic flow movement that can be followed uninterruptedly across the wall into the left ventricle. 
It was concluded that the only possibility of differentiation between a false and a true aneurysm is the angiocardiographic visualisation of a small channel connecting the left ventricle with the cavity of the pseudoaneurysm. This, however, necessitates very careful cardiac catheterisation and the use of contrast medium.

As shown in this study and by others (Roelandt et al., 1975; Davidson et al., 1977), echocardiography is a very sensitive tool for demonstrating fluid-filled spaces behind the heart. This, and the unique ability of echocardiography to differentiate between soft tissue layers such as epicardium, pericardium, and myocardium and the ease of use make this non-invasive method very suitable.

Although $\mathrm{M}$-mode echocardiography can detect and localise echo-free areas, two-dimensional echocardiography adds additional information because of the spatial orientation of the individual echoline in several examination planes. Thus size, shape, location, and extension of fluid-filled spaces and their respective walls can be shown with greater accuracy.

Despite these advantages, however, echocardiography is not reliable in differentiating between pseudoaneurysms, localised pericardial effusions, pericardial diverticula, large haematomas, or true aneurysms because beam width properties do not allow the demonstration of small-sized communication defects.

Definite diagnosis could only be made by angiocardiography. With the development of pulsed Doppler echocardiography a method is inaugurated that allows the determination of blood flow characteristics, that is turbulences or smoothness, exact timing, and direction of flow in any part of the cardiovascular system accessible to the investigation by ultrasound waves.

In this particular case it was possible to demonstrate similar blood flow patterns in the left ventricle and the cavity of the echo-free space in the direct vicinity of the left ventricular posterior wall, indicating that this space is in free communication with the ventricular cavity. Pericardial effusions, diverticula, or haematomas that have separating walls and no communication show no flow patterns within their cavities.

Differentiation between a false and a true aneurysm could be made by showing high systolic turbulence across the left ventricular posterior wall at the communication site and no flow movement in the middle and posterior part of the pseudoaneurysm.

This indicates a small restrictive defect that does not allow propagation of left ventricular pulsatile flow deeper into the cavity.

We conclude that pulsed Doppler echocardiography in combination with $\mathrm{M}$-mode and twodimensional echocardiography presents a safe and sensitive diagnostic method of detecting left ventricular aneurysms and in differentiating between false and true aneurysms.

\section{References}

Baker, D. W., Rubenstein, S. A., and Lorch, S. G. (1977). Pulsed Doppler echocardiography: principles and applications. American fournal of Medicine, 63, 69-80.

Botvinick, E. H., Shames, D., Hutchinson, J. C., Roe, B. B., and Fitzpatrick, M. (1976). Non-invasive diagnosis of a false left ventricular aneurysm with radioisotope gated cardiac blood pool imaging. American fournal of Cardiology, 37, 1089-1093.

Davidson, K. H., Parisi, A. F., Harrington, J. J., Barsamian, E. M., and Fishbein, M. C. (1977). Pseudoaneurysm of the left ventricle: an unusual echocardiographic presentation. Annals of Internal Medicine, 86, 430-433.

Gueron, M., Wanderman, K. L., Hirsch, M., and Borman, J. (1975). Pseudoaneurysms of the left ventricle after myocardial infarction. Fournal of Thoracic and Cardiovascular Surgery, 69, 736-742.

Hurst, C. O., Fine, G., and Keyes, J. W. (1963). Pseudoaneurysms of the heart. Report of a case and review of literature. Circulation, 28, 427-436.

Roelandt, J., van dem Brand, M., Vletter, W. B., Nauta, J., and Hugenholtz, P. G. (1975). Echocardiographic diagnosis of pseudoaneurysms of the left ventricle. Circulation, 52, 466-472.

Van Mechelen, R., van Hemel, N. M., and van Rijk, P. P. (1978). Noninvasive diagnosis of pseudoaneurysm of the left ventricle. British Heart fournal, 40, 812-816.

Van Tassel, H., and Edwards, J. W. (1972). Rupture of heart complicating myocardial infarction. Chest, 61, 104-116.

Vlodaver, Z., Coe, J. I., and Edwards, J. E. (1975). True and false left ventricular aneurysms, clinicopathologic correlations. Circulation, 51, 567-572.

Requests for reprints to Dr E Grube, Medizinische Universitätsklinik, D-5300 Bonn, West Germany. 